\title{
AN EHEALTH ADOPTION FRAMEWORK FOR DEVEloping CounTRIES: A SYSTEMATIC REVIEW
}

\author{
Agnes Rwashana Semwanga, Hasifah Kasujja Namatovu, Swaib Kyanda \\ Kaawaase and Mark Abraham Magumba
}

College of Computing and Information Sciences, Makerere University, Kampala, Uganda

\begin{abstract}
There is growing interest in the rate of eHealth uptake resulting from the increased potential to advance the quality of healthcare services in both the developed and developing countries. Although the implementation of information and communication technology to support healthcare delivery would greatly address the quality and accessibility challenges in healthcare as well as reduction in the cost of healthcare delivery, the adoption of eHealth has not been fully realized. This study aimed at conducting a systematic literature review to establish the factors associated with the adoption of eHealth and propose a context-specific framework for successful adoption of eHealth technologies in developing countries such as Uganda. The systematic literature review process was guided by the Systematic Review Protocol. The review of 29 journals from the period 2009-2021 showed that, although the most widely used frameworks in the developing countries were Technology Adoption Model (TAM), Unified Theory of Acceptance and Use of Technology (UTAUT) framework and Technology Organization Environment (TOE) framework, there were other salient factors reported by other researchers that contributed to the adoption of eHealth in developing countries. A novel framework for adoption of eHealth in the local context with eight (8) dimensions namely; Socio-demographic, Technology, Information, Socio-cultural, Organization, Governance, Ethical and legal and Financial dimensions is derived and presented as result of the research.
\end{abstract}

\section{KEYWORDS}

eHealth, mHealth, adoption, frameworks, developing country

\section{INTRODUCTION}

eHealth is the delivery of health services to patients who are not in direct contact with the healthcare providers through electronic means by using a variety of modern electronic information and communication technologies. [1] and [2] define eHealth as an umbrella term that covers a wide range of health and care services delivered through ICTs such as electronic health records, health information systems, remote monitoring and consultation services (telehealth, telemedicine, telecare), tools for self-management, and health data analytics. mHealth is a subcategory of eHealth that is connected to mobile telephony and applications. Research has shown that there is a growing interest in eHealth uptake and use resulting from the increased knowledge of the potential to advance the quality of healthcare services in both the developed and developing countries [3], [4]. The implementation of information and communication technology to support healthcare delivery is a key driver in addressing the quality and accessibility challenges in healthcare as well as reduction in the cost of healthcare delivery [5]. Although there is increased use of the eHealth implementations, the high failure rates often resulting from the lack of eHealth readiness have been reported in both the developed and developing countries. eHealth implementation could be of more value to health systems in lowand middle-income countries (LMICs) however, there are barriers to such implementations such 
as; limited resources and lack of competent workforce to develop and maintain such eHealth systems. Healthcare institutions or communities need to be adequately prepared if the anticipated change brought by programs related to information and communication technologies is to be realized.

Several studies indicate a slow progress in the adoption of eHealth in Sub Saharan Africa which is partly caused by primary user's limited computer skills and poor internet connectivity, poor existing infrastructure and network failure lack of prior planning for example strategy and need readiness assessment and lack of eHealth policy and strategy [1]-[5]. In a commitment to embrace technology to augment healthcare delivery, Ghana reported that very little was known about the characteristics of the required workforce and yet the country's eHealth strategy could not be fully realized without a well-trained workforce [6], [7], [8]. The research further mentioned the critical need to identify the essential numbers of the eHealth staff categories and the training needs necessary for effective and efficient adoption of eHealth. The adoption and implementation of EHR at 646 sites in Kenya showed variable dictionaries across implementations resulting into inability to easily share data across systems, lack of expertise in dictionary management, lack of central coordination and custody of a terminology service, inadequately defined policies and processes, insufficient infrastructure, among others [9].

Although Uganda has shown a growing increase in the use of eHealth and m-Health applications as a means to improve healthcare service delivery, their success is far below expectation [4]. The health eco-system in Uganda is largely operating on silo systems with large appreciation of eHealth in improving service delivery but with less enforcement and adoption of the same [4], [10]. There is a need for a structured approach to assess the status of eHealth technology adoption and subsequently establish where they have failed and why.

There has been great investment in the sector at individual, national, regional and international level, however there is little appreciation and adoption of the same. Alaboudi et al., [11] noted that there was neither one-size-fit-all framework nor best-practice solution for all ICT innovations or for all countries while each country / organisation experienced barriers and challenges in adopting and implementing one ICT innovation. Several eHealth adoption models and frameworks such as the "Fit between Individuals, Task and Technology" (FITT) framework, Technology Adoption Model (TAM), Diffusion of Innovations Theory and Theory of Acceptance and Use Technology (UTAUT) have been developed by several researchers, however, there is still need to establish why these have not yielded the anticipated results [12], [13], [14], [15]. [12]-[15]

Although, several adoption theories and models have been proposed in the past, there is still need to identify a context-specific framework for successful adoption of eHealth technologies in developing countries. There's a huge disparity in the socio-economic, socio-cultural, sociodemographic and political setting between the developed and developing countries, yet eHealth adoption has been treated as a "one-size-fits-all." Hence, the purpose of this study was to conduct a systematic review to establish key factors associated with the adoption of eHealth in developing countries and subsequently develop a framework that fits the context of developing countries. like Uganda.

\section{The Systematic Literature RevieW}

The main reason for conducting the systematic literature review was to establish the factors to be used to identify and propose an eHealth adoption framework for a developing country like 
Uganda. The systematic review followed the protocol by [16], highly acknowledged in Software Engineering as shown in Table 1.

Table 1: Systematic Review Protocol (adopted from [16])

\begin{tabular}{|l|l|}
\hline Background & $\begin{array}{l}\text { Several adoption theories and models have been proposed, however, the } \\
\text { adoption of eHealth has not yielded the anticipated results. The success of } \\
\text { eHealth and m-Health applications towards the improvement of healthcare } \\
\text { service delivery is far below expectation. There is need to establish a } \\
\text { suitable framework that will guide the adoption of eHealth in the Ugandan } \\
\text { context. }\end{array}$ \\
\hline Research Question & $\begin{array}{l}\text { What factors are essential for the development of a context-specific } \\
\text { framework for successful adoption of eHealth technologies in Uganda? }\end{array}$ \\
\hline Strategy & $\begin{array}{l}\text { Sources included the following databases: Science Direct, PubMed, } \\
\text { ProQuest, Google Scholar, Elsevier. } \\
\text { Keywords: "eHealth" AND "Adoption Frameworks", AND "Developing } \\
\text { Countries", "eHealth" AND "Acceptance", "Telemedicine" AND } \\
\text { "Adoption", "Telemedicine" AND "Acceptance". }\end{array}$ \\
\hline Study selection criteria & $\begin{array}{l}\text { Journal articles related to the adoption of eHealth published from 2009 to } \\
\text { 2021. Three search types of logical criterion in the search field: } \\
1 . \quad \text { Search Type 1 : Title field } \\
2 . \quad \text { Search Type 2: Title field, Abstract and Keywords field } \\
\text { 3. Search Type 3: All fields }\end{array}$ \\
\hline $\begin{array}{l}\text { Study selection } \\
\text { procedures }\end{array}$ & $\begin{array}{l}\text { Journal articles that contain any kind of review, evaluation or assessment } \\
\text { about eHealth system(s) }\end{array}$ \\
\hline Data extraction strategy & $\begin{array}{l}\text { Data was extracted from each journal paper as follows: Source of the } \\
\text { journal, year, when the paper was published, aim of the study, the country, } \\
\text { study design, framework or models used and factors/ variables affecting } \\
\text { adoption,. }\end{array}$ \\
\hline
\end{tabular}

\section{The Methodology And FindingS}

The Systematic Literature Review (SLR) was conducted which involved three phases, namely; i) planning the review, ii) conducting the review and iii) reporting the review as suggested by [17].

\subsection{Planning the Review}

The planning started with the drafting of the review protocol which was shared with the four members of the research team during a brainstorming session. The protocol was examined to ensure that it was aligned with the goals of the study and the research questions. The protocol was further examined to ensure that the selected key words were derived from the research questions and that the data extracted and analysis would address the research question.

\subsection{Conducting the Review}

The search with the key words was run for each logical criterion in the search field and was further reduced according to the following inclusion criteria:

a. The journal paper must have been published from 2009, since 12 years was considered adequate time to examine the eHealth adoption in developing countries where e-Health adoption is still in the infancy stage.

b. The focus of the journal paper was the eHealth adoption in developing countries.

c. The journal paper must have been published in an international journal of interest. 
The first step of the review process involved carrying out preliminary searches of the systematic review papers to establish those that were found relevant. Secondly, a web search was performed to find out other relevant concepts. Several sets of key words were used to combine the concepts of the study such as "eHealth" AND "Adoption Frameworks" AND "Developing Countries", "eHealth" AND "Acceptance", "Telemedicine" AND "Adoption", "Telemedicine" AND "Acceptance" and "eHealth" and "Readiness" and "eHealth" and "Assessment". Additionally, articles in the reference lists of the selected studies were used to identify more concepts to enhance the review.

\subsection{Reporting the Review}

A search in Pubmed for (Adoption Framework) AND (eHealth) AND (Developing country) restricted to journal articles from 2009 to 2021 resulted in 18 publications which were identified and characterized, and the more relevant articles analyzing eHealth adoption frameworks in LMIC from a more generic perspective were analyzed in detail. A similar search on Google Scholar resulted in 43 publications, while searches on Science Direct, Elsevier and ProQuest did not yield any results. There were 61 database searches. The inclusion criteria were met by only 29 articles which were included in the study.

\subsection{Findings}

The papers that were reviewed examined the factors associated with the access, influence, culture, reinforcement, acceptance and adoption of eHealth as well as the barriers, challenges, enablers, inhibitors and outcomes of eHealth adoption. The methods in the study design (reviewed papers) included field surveys, semi-structured and structured questionnaires, interviews, participant observations, electronic literature search and reviews, concept working groups, mixed methods, case studies and fuzzy analytic network process among others. The table below presents the authors, aim of the study, country, study design, frameworks and models used and factors affecting adoption as reported by the 29 papers that were reviewed.

Table 2: Review of eHealth Adoption Frameworks / Models

\begin{tabular}{|c|c|c|c|c|c|}
\hline $\begin{array}{l}\text { Aut } \\
\text { hor }\end{array}$ & Aim & $\begin{array}{c}\text { Count } \\
\text { ry }\end{array}$ & $\begin{array}{l}\text { Study } \\
\text { Design }\end{array}$ & $\begin{array}{l}\text { Framework / } \\
\text { Models used }\end{array}$ & $\begin{array}{c}\text { Factors / Variables affecting } \\
\text { adoption }\end{array}$ \\
\hline [18] & $\begin{array}{l}\text { Study the factors } \\
\text { affecting the } \\
\text { adoption and } \\
\text { acceptance of e- } \\
\text { Health in the } \\
\text { context of Saudi } \\
\text { Arabia }\end{array}$ & $\begin{array}{l}\text { Saudi } \\
\text { Arabi } \\
\text { a }\end{array}$ & $\begin{array}{l}\text { Survey } \\
\text { data }\end{array}$ & $\begin{array}{l}\text { Technology } \\
\text { Acceptance } \\
\text { Model (TAM) } \\
\text { and Theory of } \\
\text { Planned } \\
\text { Behavior } \\
\text { frameworks } \\
\text { (TPB). }\end{array}$ & $\begin{array}{l}\text { Significant influence of } \\
\text { perceived usefulness and } \\
\text { perceived ease of use on the } \\
\text { attitude. } \\
\text { Attitude and subjective norm } \\
\text { significantly influence patient } \\
\text { behavioral intention to use e- } \\
\text { health services. }\end{array}$ \\
\hline [19] & $\begin{array}{l}\text { Investigate the } \\
\text { influence of culture } \\
\text { on the adoption of } \\
\text { e-Health in } \\
\text { Bangladesh. }\end{array}$ & $\begin{array}{l}\text { Bangl } \\
\text { adesh }\end{array}$ & $\begin{array}{l}\text { Structure } \\
\mathrm{d} \\
\text { question } \\
\text { naire }\end{array}$ & $\begin{array}{l}\text { Hofstede's } \\
\text { cultural } \\
\text { dimension } \\
\text { model and the } \\
\text { Technology } \\
\text { Acceptance } \\
\text { Model (TAM). }\end{array}$ & $\begin{array}{l}\text { Cultural dimensions such as } \\
\text { power, distance, masculinity, } \\
\text { restraint and perceived } \\
\text { usefulness had significant } \\
\text { impacts on intention to use } \\
\text { eHealth. }\end{array}$ \\
\hline
\end{tabular}


Health Informatics - An International Journal (HIIJ) Vol.10, No.3, August 2021

\begin{tabular}{|c|c|c|c|c|c|}
\hline [12] & $\begin{array}{l}\text { Analyze the socio- } \\
\text { organizational- } \\
\text { technical factors } \\
\text { that influence IT } \\
\text { adoption in the } \\
\text { healthcare }\end{array}$ & Crete & $\begin{array}{l}\text { Intervie } \\
\text { ws and } \\
\text { participa } \\
\text { nt } \\
\text { observati } \\
\text { ons }\end{array}$ & $\begin{array}{l}\text { "Fit between } \\
\text { Individuals, } \\
\text { Task and } \\
\text { Technology" } \\
\text { (FITT) } \\
\text { framework } \\
\text { domain. }\end{array}$ & $\begin{array}{l}\text { Organizational factors that } \\
\text { influence IT adoption in } \\
\text { healthcare- Fit management, fit } \\
\text { between individuals, task and } \\
\text { technology }\end{array}$ \\
\hline [13] & $\begin{array}{l}\text { To explore the } \\
\text { factors that } \\
\text { influence rural end } \\
\text { users' acceptance of } \\
\text { ehealth in } \\
\text { Bangladesh. }\end{array}$ & $\begin{array}{l}\text { Bangl } \\
\text { adesh }\end{array}$ & $\begin{array}{l}\text { Field } \\
\text { survey, } \\
\text { structure } \\
\text { d } \\
\text { question } \\
\text { naire and } \\
\text { Logistic } \\
\text { regressio } \\
\text { n } \\
\text { analysis. }\end{array}$ & $\begin{array}{l}\text { Technology } \\
\text { Acceptance } \\
\text { Model (TAM) } \\
\text { was adopted as } \\
\text { the research } \\
\text { framework. }\end{array}$ & $\begin{array}{l}\text { Influential variables were social } \\
\text { reference, advertisement, } \\
\text { attitude toward the system, } \\
\text { access to cell phone and } \\
\text { perceived system } \\
\text { effectiveness. Significant } \\
\text { demographic variables on } \\
\text { eHealth acceptance behavior } \\
\text { were, age, gender, and } \\
\text { education. }\end{array}$ \\
\hline [20] & $\begin{array}{l}\text { Explore the need to } \\
\text { positively reinforce } \\
\text { the adoption of } \\
\text { telemedicine } \\
\text { amongst clinicians } \\
\text { in Nigeria. }\end{array}$ & $\begin{array}{l}\text { Nigeri } \\
\mathrm{a}\end{array}$ & $\begin{array}{l}\text { Structure } \\
\mathrm{d} \text { paper- } \\
\text { based } \\
\text { question } \\
\text { naires }\end{array}$ & $\begin{array}{l}\text { Unified Theory } \\
\text { of Acceptance } \\
\text { and Use of } \\
\text { Technology } \\
\text { (UTAUT) } \\
\text { model }\end{array}$ & $\begin{array}{lr}\text { Influential factors were } \\
\text { performance expectancy, effort } \\
\text { expectancy, racilitating } \\
\text { condition and reinforcement } \\
\text { determinants. }\end{array}$ \\
\hline [21] & $\begin{array}{l}\text { Explore } r \text { and } \\
\text { analyze the current } \\
\text { level of, reasons } \\
\text { behind and factors } \\
\text { affecting consumer } \\
\text { acceptance r of } \\
\text { Portable Health } \\
\text { Clinic (PHC). }\end{array}$ & $\begin{array}{l}\text { Bangl } \\
\text { adesh }\end{array}$ & $\begin{array}{l}\text { Qualitati } \\
\text { ve and } \\
\text { explorat } \\
\text { ory } \\
\text { survey } \\
\text { among } \\
600 \\
\text { inhabitan } \\
\text { ts }\end{array}$ & $\begin{array}{l}\text { Technology } \\
\text { Acceptance } \\
\text { Model (TAM) }\end{array}$ & $\begin{array}{l}\text { Consumer's age, occupation } \\
\text { and purchasing power have } \\
\text { very strong influence on their } \\
\text { acceptance of eHealth services } \\
\text { while their gender, level of } \\
\text { education, access to cell phone } \\
\text { and previous eHealth } \\
\text { knowledge have very } \\
\text { insignificant or weaker impact. }\end{array}$ \\
\hline$[22]$ & $\begin{array}{l}\text { Review published } \\
\text { research on TAM } \\
\text { use in health } \\
\text { information systems } \\
\text { development and } \\
\text { implementation with } \\
\text { regard to application } \\
\text { areas and model } \\
\text { extensions after its } \\
\text { initial introduction. }\end{array}$ & $\begin{array}{l}\text { Divers } \\
\mathrm{e}\end{array}$ & $\begin{array}{l}\text { Electroni } \\
\text { c } \\
\text { literature } \\
\text { search } \\
\text { from } 134 \\
\text { articles }\end{array}$ & $\begin{array}{l}\text { Technology } \\
\text { Acceptance } \\
\text { Model (TAM) }\end{array}$ & $\begin{array}{l}\text { The review identified three } \\
\text { main information and } \\
\text { communication technology } \\
\text { (ICT) application areas for the } \\
\text { TAM in health services: } \\
\text { telemedicine, electronic health } \\
\text { records, and mobile } \\
\text { applications }\end{array}$ \\
\hline
\end{tabular}


Health Informatics - An International Journal (HIIJ) Vol.10, No.3, August 2021

\begin{tabular}{|c|c|c|c|c|c|}
\hline [23] & $\begin{array}{l}\text { Explore the factors } \\
\text { that affect } \\
\text { individuals' } \\
\text { acceptance of using } \\
\text { social media as a } \\
\text { tool for receiving } \\
\text { health awareness }\end{array}$ & $\begin{array}{l}\text { Saudi } \\
\text { Arabi } \\
\text { a, the } \\
\text { Unite } \\
\text { d } \\
\text { Kingd } \\
\text { om, } \\
\text { and } \\
\text { the } \\
\text { Unite } \\
\text { d } \\
\text { Arab } \\
\text { Emira } \\
\text { tes }\end{array}$ & Survey & $\begin{array}{l}\text { Technology } \\
\text { Acceptance } \\
\text { Model (TAM), } \\
\text { Task } \\
\text { Technology Fit } \\
\text { (TTF) and } \\
\text { Prospect Theory } \\
\text { resulting into } \\
\text { Smart Health } \\
\text { Awareness } \\
\text { Message Framework }\end{array}$ & $\begin{array}{l}\text { Perceived usefulness } \\
\text { significantly affects people } \\
\text { toward social media adaptation } \\
\text { in sharing health information. } \\
\text { Perceptions of the benefits of } \\
\text { adopting health messages, the } \\
\text { design of health messages, } \\
\text { patient friendly and } \\
\text { technologically materials } \\
\text { encouraged individuals to } \\
\text { exchange these messages } \\
\text { among social media users. }\end{array}$ \\
\hline [11] & $\begin{array}{l}\text { Identify the } \\
\text { principle predictive } \\
\text { challenges and } \\
\text { barriers in the } \\
\text { context of the } \\
\text { Kingdom of Saudi } \\
\text { Arabia and } \\
\text { understand the } \\
\text { perspective of the } \\
\text { decision makers of } \\
\text { each HCF type, } \\
\text { sector, and location. }\end{array}$ & $\begin{array}{l}\text { Kingd } \\
\text { om of } \\
\text { Saudi } \\
\text { Arabi } \\
\text { a }\end{array}$ & $\begin{array}{l}\text { Literatur } \\
\text { e review, } \\
\text { intervie } \\
\text { ws, and } \\
\text { question } \\
\text { naires }\end{array}$ & $\begin{array}{l}\text { Unified Theory } \\
\text { of Acceptance } \\
\text { and Use of } \\
\text { Technology } \\
\text { (UTAUT), the } \\
\text { Technology, } \\
\text { Organisation } \\
\text { and } \\
\text { Environment } \\
\text { (TOE) } \\
\text { theoretical } \\
\text { framework, and } \\
\text { the Evaluating } \\
\text { Telemedicine } \\
\text { Systems } \\
\text { Success Model } \\
\text { (ETSSM). }\end{array}$ & $\begin{array}{l}\text { Barriers to adoption of } \\
\text { telemedicine services by } \\
\text { healthcare facilities are (i) the } \\
\text { availability of adequate } \\
\text { sustainable financial support to } \\
\text { implement, operate, and } \\
\text { maintain the telemedicine } \\
\text { system, (ii) ensuring } \\
\text { conformity of telemedicine } \\
\text { services with core mission, } \\
\text { vision, needs and constraints of } \\
\text { the HCF, and (iii) the } \\
\text { reimbursement for telemedicine } \\
\text { services. }\end{array}$ \\
\hline [9] & $\begin{array}{lr}\text { Examine } & \text { policies } \\
\text { and } & \text { processes } \\
\text { around dictionary } \\
\text { management }\end{array}$ & Kenya & $\begin{array}{l}\text { Concept } \\
\text { working } \\
\text { group }\end{array}$ & $\begin{array}{l}\text { Framework for } \\
\text { maintenance of } \\
\text { medical } \\
\text { terminological } \\
\text { systems by } \\
\text { Bakhshi-Raiez } \\
\text { et al. (2008). }\end{array}$ & $\begin{array}{l}\text { Policies and processes around } \\
\text { dictionary management }\end{array}$ \\
\hline [24] & $\begin{array}{l}\text { Investigate the } \\
\text { factors that affect } \\
\text { the adoption of } \\
\text { mHealth services in } \\
\text { Bangladesh using } \\
\text { Extended } \\
\text { Technology } \\
\text { Acceptance Model. }\end{array}$ & $\begin{array}{l}\text { Bangl } \\
\text { adesh }\end{array}$ & Survey & $\begin{array}{l}\text { Extended } \\
\text { Technology } \\
\text { Acceptance } \\
\text { Model (TAM). }\end{array}$ & $\begin{array}{l}\text { Adoption of a system such as } \\
\text { mHealth is dependent on } \\
\text { usefulness of the systems and } \\
\text { ease of use. }\end{array}$ \\
\hline [25] & $\begin{array}{l}\text { Identify relevant } \\
\text { elements of Internet } \\
\text { adoption at user } \\
\text { level }\end{array}$ & $\begin{array}{l}\text { Gamb } \\
\text { ia }\end{array}$ & $\begin{array}{l}\text { Question } \\
\text { naires }\end{array}$ & $\begin{array}{l}\text { Unified Theory } \\
\text { Acceptance and } \\
\text { Use of } \\
\text { Technology } \\
\text { (UTAUT) }\end{array}$ & $\begin{array}{l}\text { Direct determinants: education, } \\
\text { behavioural intention and } \\
\text { income. Indirect determinants: } \\
\text { performance expectancy, effort } \\
\text { expectancy, social influence } \\
\text { and facilitating conditions. } \\
\text { Moderating factors: age, } \\
\text { gender, experience, voluntary } \\
\text { use, friends' influence }\end{array}$ \\
\hline
\end{tabular}


Health Informatics - An International Journal (HIIJ) Vol.10, No.3, August 2021

\begin{tabular}{|c|c|c|c|c|c|}
\hline [26] & $\begin{array}{l}\text { Review and } \\
\text { document patient } \\
\text { adoption issues and } \\
\text { develop } \\
\text { framework. }\end{array}$ & $\begin{array}{l}\text { Devel } \\
\text { oping } \\
\text { countr } \\
\text { ies } \\
\text { South } \\
\text { Africa }\end{array}$ & $\begin{array}{l}\text { Literatur } \\
\text { e search }\end{array}$ & $\begin{array}{l}\text { Patient m-health } \\
\text { Adoption Frame }\end{array}$ & $\begin{array}{l}\text { Socio cultural practices, } \\
\text { awareness of eHealth and } \\
\text { impacted adoption, high and } \\
\text { unrealistic expectations and } \\
\text { mHealth, access to mobile } \\
\text { devices, accessible power, } \\
\text { mobile network service } \\
\text { coverage and language } \\
\text { behavior }\end{array}$ \\
\hline [27] & $\begin{array}{l}\text { Understand } \\
\text { potential factors } \\
\text { driving / inhibiting } \\
\text { the decision of HIS } \\
\text { adoption in } \\
\text { Malaysia }\end{array}$ & $\begin{array}{l}\text { Malay } \\
\text { sia }\end{array}$ & $\begin{array}{l}\text { Fuzzy } \\
\text { analytic } \\
\text { network } \\
\text { process } \\
\text { and } \\
\text { survey }\end{array}$ & $\begin{array}{l}\text { Integrated } \\
\text { Diffusion of } \\
\text { Innovation } \\
\text { Theory (DOI), } \\
\text { Technology, } \\
\text { Organisation } \\
\text { Environment } \\
\text { (TOE) } \\
\text { framework, } \\
\text { Human } \\
\text { Organisation } \\
\text { Technology Fit. }\end{array}$ & $\begin{array}{l}\text { Decision to adopt HIS mainly } \\
\text { determined by technological } \\
\text { and environment context. }\end{array}$ \\
\hline [28] & $\begin{array}{l}\text { Establish the } \\
\text { determinants } \\
\text { affecting the } \\
\text { adoption of master } \\
\text { data management }\end{array}$ & $\begin{array}{l}\text { Malay } \\
\text { sia }\end{array}$ & $\begin{array}{l}\text { Empirica } \\
\text { 1 study }\end{array}$ & $\begin{array}{l}\text { Technology } \\
\text { Organisation } \\
\text { Environment } \\
\text { (TOE) } \\
\text { framework }\end{array}$ & $\begin{array}{l}\text { Top management support, } \\
\text { relative advantage, data security } \\
\text { and government policy. }\end{array}$ \\
\hline [29] & $\begin{array}{l}\text { Identify factors } \\
\text { influencing the } \\
\text { adoption of HIS in } \\
\text { public hospitals }\end{array}$ & $\begin{array}{l}\text { Pakist } \\
\text { an }\end{array}$ & $\begin{array}{l}\text { Question } \\
\text { naires, } \\
\text { Confirm } \\
\text { atory } \\
\text { Factor } \\
\text { Analysis } \\
\text { and } \\
\text { Structure } \\
\text { Equation } \\
\text { Modelin } \\
\text { g were } \\
\text { applied. }\end{array}$ & $\begin{array}{l}\text { Framework for } \\
\text { the adoption of } \\
\text { HIS in public } \\
\text { sector hospitals } \\
\text { of Pakistan }\end{array}$ & $\begin{array}{l}\text { Top management, financial } \\
\text { revenue, relative advantage, } \\
\text { compatibility, coercive pressure } \\
\text { and mimetic pressure positively } \\
\text { influenced HIS adoption. }\end{array}$ \\
\hline [30] & $\begin{array}{l}\text { Study the adoption } \\
\text { of an integrated } \\
\text { hospital information } \\
\text { system }\end{array}$ & $\begin{array}{l}\text { Ugand } \\
\text { a }\end{array}$ & $\begin{array}{l}\text { Intervie } \\
\text { ws, } \\
\text { question } \\
\text { naires }\end{array}$ & UTAUT & $\begin{array}{l}\text { Factors that } \begin{array}{r}\text { enhanced use } \\
\text { included; }\end{array} \text { expected } \\
\text { improvement in job } \\
\text { performance, easiness of } \\
\text { learning and using systems, } \\
\text { support and influence from } \\
\text { management and peers, } \\
\text { availability of organizational } \\
\text { and technical infrastructure. }\end{array}$ \\
\hline [31] & $\begin{array}{l}\text { Comprehend several } \\
\text { factors affecting } \\
\text { adoption of cloud } \\
\text { computing in } \\
\text { healthcare } \\
\text { organisations }\end{array}$ & Jordan & $\begin{array}{l}\text { Quantitat } \\
\text { ive } \\
\text { approach } \\
\text {, surveys }\end{array}$ & $\begin{array}{l}\text { Technology, } \\
\text { Organisation } \\
\text { and } \\
\text { Environment } \\
\text { (TOE) }\end{array}$ & $\begin{array}{l}\text { Technology, organization and } \\
\text { environment are important and } \\
\text { could have an impact of cloud } \\
\text { computing. }\end{array}$ \\
\hline
\end{tabular}


Health Informatics - An International Journal (HIIJ) Vol.10, No.3, August 2021

\begin{tabular}{|c|c|c|c|c|c|}
\hline [32] & $\begin{array}{l}\text { Examine the } \\
\text { relationships of } \\
\text { health practitioners } \\
\text { intentions to adopt } \\
\text { eHealth systems. }\end{array}$ & $\begin{array}{l}\text { Thaila } \\
\text { nd }\end{array}$ & $\begin{array}{l}\text { Quantitat } \\
\text { ive }\end{array}$ & $\begin{array}{l}\text { Used constructs } \\
\text { of belief, } \\
\text { attitude and } \\
\text { intentions. }\end{array}$ & $\begin{array}{l}\text { Beliefs, intentions are higher on } \\
\text { attitudes. }\end{array}$ \\
\hline [37] & $\begin{array}{l}\text { Analyses the } \\
\text { technology adoption } \\
\text { framework to } \\
\text { determine a suitable } \\
\text { model }\end{array}$ & $\begin{array}{l}\text { South } \\
\text { Africa }\end{array}$ & $\begin{array}{l}\text { Survey, } \\
\text { question } \\
\text { naires, } \\
\text { quantitat } \\
\text { ive } \\
\text { approach } \\
\text { statistica } \\
1 \\
\text { Cronbac } \\
\text { h Alpha } \\
\text { and } \\
\text { multi- } \\
\text { linear } \\
\text { regressio } \\
\text { n }\end{array}$ & & $\begin{array}{l}\text { Usefulness, ease of use, } \\
\text { knowledge of electronic patient } \\
\text { record management system, } \\
\text { ICT infrastructure, technology } \\
\text { readiness, organizational } \\
\text { readiness, top management } \\
\text { support and compatibility of } \\
\text { electronic patient record } \\
\text { management systems. }\end{array}$ \\
\hline [34] & $\begin{array}{l}\text { Examine the factors } \\
\text { influencing adoption } \\
\text { of the computer } \\
\text { assisted medical } \\
\text { diagnosis from } \\
\text { Kenyan perspective }\end{array}$ & Kenya & $\begin{array}{l}\text { Data } \\
\text { collectio } \\
\mathrm{n} \quad \text { and } \\
\text { statistica } \\
1 \\
\text { analysis }\end{array}$ & $\begin{array}{l}\text { Reviewed } \\
\text { several theories; } \\
\text { UTAUT, } \\
\text { Theory of } \\
\text { Reasoned Act, } \\
\text { Theory of } \\
\text { Planned } \\
\text { Behaviour }\end{array}$ & $\begin{array}{l}\text { How gender, age and specialty } \\
\text { influence these constructs; } \\
\text { perceived usefulness, perceived } \\
\text { ease of use, perceived } \\
\text { information quality, perceived } \\
\text { trust, security, awareness, ICT } \\
\text { skills and savings / cost as } \\
\text { constructs and user } \\
\text { satisfaction. }\end{array}$ \\
\hline [35] & $\begin{array}{l}\text { Identify essential } \\
\text { factors that are } \\
\text { significant driving } \\
\text { or hindering the } \\
\text { decision to adopt } \\
\text { HIS. }\end{array}$ & $\begin{array}{l}\text { Malay } \\
\text { sia }\end{array}$ & $\begin{array}{l}\text { Decision } \\
\text { Making } \\
\text { Trial and } \\
\text { Evaluati } \\
\text { on Lab, } \\
\text { Analytic } \\
\text { Network } \\
\text { Process } \\
\text { Method } \\
\text { to assess } \\
\text { depende } \\
\text { ncy }\end{array}$ & $\begin{array}{l}\text { Technology } \\
\text { Organisation } \\
\text { Environment } \\
\text { (TOE) }\end{array}$ & $\begin{array}{l}\text { Technical competence, } \\
\text { compatibility, top management } \\
\text { support, vendor support were } \\
\text { the most essential for } \\
\text { successful adoption of HIS. }\end{array}$ \\
\hline [36] & $\begin{array}{l}\text { The aim of this } \\
\text { study was to } \\
\text { evaluate key } \\
\text { informant (local } \\
\text { expert) opinions on } \\
\text { aspects that need to } \\
\text { be considered when } \\
\text { developing an } \\
\text { eHRA framework } \\
\text { suitable for use in } \\
\text { developing } \\
\text { countries. }\end{array}$ & $\begin{array}{l}\text { Botsw } \\
\text { ana }\end{array}$ & $\begin{array}{l}\text { Intervie } \\
\text { ws }\end{array}$ & $\begin{array}{l}\text { eHealth } \\
\text { Readiness } \\
\text { Assessment } \\
\text { Framework }\end{array}$ & 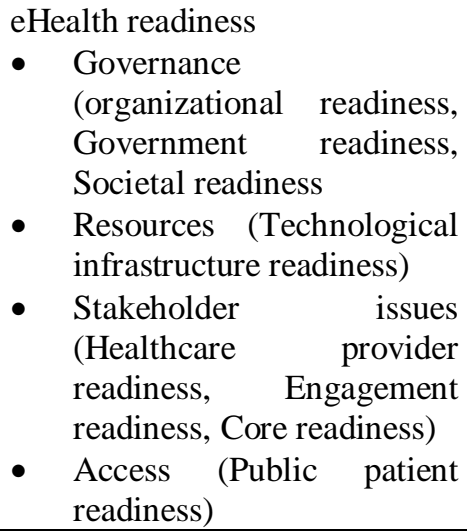 \\
\hline
\end{tabular}


Health Informatics - An International Journal (HIIJ) Vol.10, No.3, August 2021

\begin{tabular}{|c|c|c|c|c|c|}
\hline [6] & $\begin{array}{l}\text { The objective of this } \\
\text { study was to } \\
\text { develop a } \\
\text { framework to } \\
\text { characterize and } \\
\text { assess the eHealth } \\
\text { workforce of } \\
\text { hospitals in LMICs. }\end{array}$ & Ghana & $\begin{array}{l}\text { Semi- } \\
\text { structure } \\
\text { d } \\
\text { intervie } \\
\text { ws }\end{array}$ & $\begin{array}{l}\text { Framework to } \\
\text { characterize and } \\
\text { assess the } \\
\text { eHealth } \\
\text { workforce in } \\
\text { LMIC. }\end{array}$ & $\begin{array}{l}\text { ehealth workforce roles } \\
\text { required for effective } \\
\text { leadership, management and } \\
\text { support or operations } \\
\text { well-trained workforce and } \\
\text { their training needs }\end{array}$ \\
\hline
\end{tabular}

\section{REVIEW OF THE FRAMEWORKS}

Several frameworks and theories have been used to examine the adoption of eHealth in developing countries as shown in the table. Some of the frameworks that have been used include; the Human Organisation Technology Fit [27], Extended Technology Acceptance Model [24], Diffusion Innovation Theory (DOI) [27], framework with social factors, cloud security risks and benefits [27], Theory of Planned Behaviour ([18], [34]), Theory of Reasoned Act [34], Fit between Individuals Task and Technology (FITT) [12] and Patient m-Health Adoption framework ([26],[36]).

However, the research showed that the most widely used frameworks for eHealth adoption in developing countries were the Technology Acceptance Model (TAM) ([18], [13], [23], [22], [23]), the Unified Theory of Acceptance and Use of Technology (UTAUT) ([11], [20], [25], [30], [34]) and the Technology, Organisation and Environment (TOE) framework ([11], [31], [28], [31], [35]).

TAM is one of the most popular and influential models of technology acceptance designed to measure the adoption of new technology based on user attitudes. The model helps to understand how people come to admit and utilize new technologies. The model utilizes two primary factors influencing and individual's intention to use new technology, namely; perceived ease of use and perceived usefulness [37]. TAM has evolved over the years and currently has many variations. From the use of UTAUT, the following factors have been identified as being related to the adoption of eHealth. The influential factors include; performance expectancy, effort expectancy, facilitating conditions and reinforcement determinants. The direct determinants are education, behavioral intention and income and the indirect determinants are performance expectancy, social influence and facilitating conditions. The Technology Organization Environment (TOE) framework on the other hand, describes factors that influence technology adoption and its likelihood as well as the process by which a firm adopts and implements technological innovations influenced by the technological context, the organizational context, and the environmental context [38]. The study used the constructs from the three most widely used as well as other frameworks to propose a framework that was suitable for eHealth adoption in developing countries.

\section{The Proposed Framework for the Adoption of EHealth in DEVELOPING COUNTRIES}

This section presents the methods used to develop and validate the framework as well as the description of the components of the framework. 


\subsection{Preparation of the Proposed Framework}

Following the systematic review of 29 papers and the discussion of the frameworks that have been used by other researchers in developing countries, factors associated with the adoption of eHealth were selected from the three most widely used frameworks (TAM, UTAUT, TOE). The factors were combined and then categorised into eight (8) dimensions which were borrowed from existing frameworks. The dimensions that were created are Socio-demographic (13), Technology (11), Information (5), Socio-cultural (7), Organization (15), Governance (3), Ethical and legal (1), and Financial dimensions (2) and these were put together to form a framework for the adoption of eHealth in Uganda as shown in Figure 1.

The proposed framework was validated following a two tier process with subject matter experts (SME's) from both academia and the industry. The first evaluation was conducted with a team of researchers at the School of Computing and Informatics Technology, Makerere University. These SME's have a good trajectory and years of experience in developing eHealth systems. Two focus group discussions involving six participants each, were organized in parallel sessions to critically examine the proposed framework. The ulterior motive was to eliminate ambiguity, monotony and identify any misrepresentations. Feedback from this team of validators was shared with the authors, who, unanimously agreed to refine the framework as suggested. The revised framework was shared with another team of validators from the medical field (5 participants) and Ministry of Health (3 participants). This team further advised to collapse some factors under the organizational and governance dimension because some factors were over-represented. It is envisaged that the publication of this paper will lead to further revision of the framework resulting from socio-cultural, economic and political disparity of different countries.

\subsection{Description of the Framework}

This section presents the proposed eHealth adoption framework as well as provides a description of the dimensions that make up the framework as shown in Figure 1.

Socio-demographic Dimension: The socio-demographic dimension has characteristics of the population or community that should be taken into consideration as new technologies are being introduced. Socio-demographic characteristics provide guidance from the design, development, implementation and adoption of the system or technology. The socio-demographic characteristics that were found to be important in the eHealth frameworks adoption included; age, gender, education level, occupation, income, awareness of eHealth (knowledge) and access to mobile devices. Other factors related to the person's experience in use of ICTs, attitudes towards eHealth, perception of usefulness, user satisfaction, performance expectancy of eHealth and perceived trust of technology were reported to contribute significantly to the adoption of eHealth.

Socio-Cultural Dimension: The socio-cultural factors are those that have social and cultural aspects. Some of the socio-cultural factors affecting eHealth adoption that must be considered are; beliefs and influence by friends in the community, power and masculinity (what the person in charge (controls the funds) says) and the distance between user and nearest health facility. Additionally, there should be societal readiness and public-patient readiness to use the technology.

Technology Dimension: Several factors related to technology were found to be important in the adoption of eHealth in developing countries. Although these countries often experience inadequacy of resources, there is need to adequately prepare for adoption of technologies. The review of eHealth adoption frameworks yielded the following as important factors towards the adoption of eHealth in developing countries; infrastructure and technology readiness, availability 
Health Informatics - An International Journal (HIIJ) Vol.10, No.3, August 2021

of organizational and technical infrastructure (electricity, mobile network), presence of integration and interoperability standards, development of IT agendas and vendor support. Further, it was noted that adoption would be enhanced if the IT design was friendly, with ease of learning and use of the technology and the usefulness of the system could be derived. The new technologies should be compatible with the existing e-patient records management systems. Additionally, there was need to establish training needs and plan to have them resolved for successful adoption of eHealth technologies. Furthermore, the use of the eHealth technology needs to be beneficial in terms of savings and cost reduction.

Information Dimension: As far as the information generated by the eHealth technologies or systems is concerned, the following factors were found to be important. The technologies should offer patient friendly health messages that are usable among technologies for enhanced exchange especially among social media users. Information from eHealth technologies or systems should be of high quality. The eHealth technologies or systems should ensure that confidentiality and privacy of information are maintained to avoid access by fraudsters. 
Health Informatics - An International Journal (HIIJ) Vol.10, No.3, August 2021

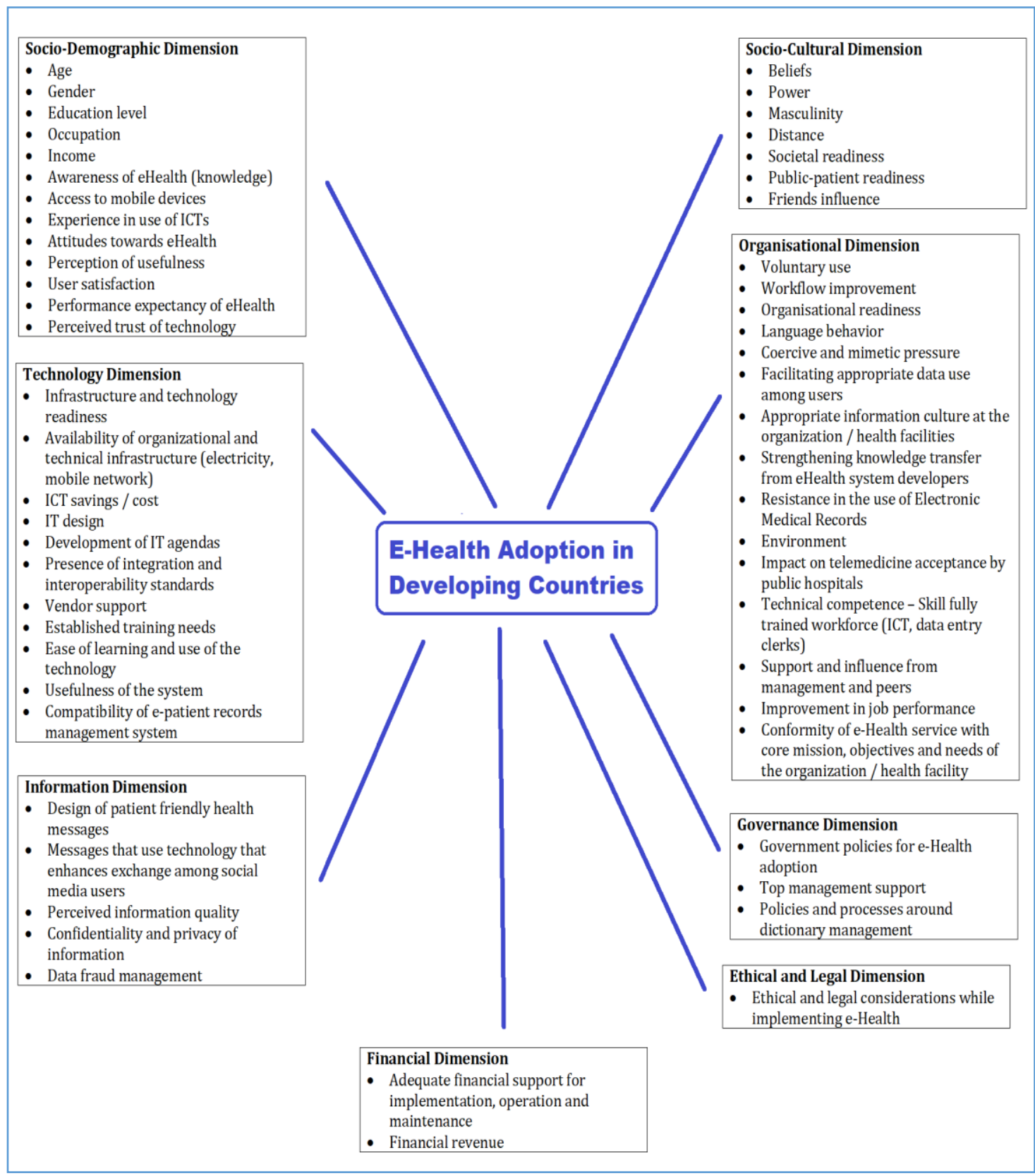

Figure 1: A Framework for eHealth Adoption in Developing Countries

Organisational Dimension: Successful eHealth adoption requires a conducive environment where there is adequate preparedness and readiness in the implementing organization / health facility. The implementation of eHealth should be in conformity with core mission, objectives and needs of the organization / health facility. There should be technical (ICT, data entry clerks) skill competence in the organization among the workforce as well as institutional and peer support/influence. Appropriate information culture at the eHealth implementing facilities and language behaviour enhances the adoption of technology. The use of coercive and mimetic pressure should be minimized and issues related to the resistance in the use of Electronic Medical Records (EMR) should be addressed while encouraging voluntary eHealth usage. The improvement of the workflows and job performance as well as strengthening knowledge transfer from eHealth system developers to users improves the adoption of eHealth. The impact of 
telemedicine acceptance by public hospitals once observed will additionally enhance the eHealth adoption.

Governance Dimension: Successful adoption of eHealth requires a high level of governance and support from top management as well as the design and implementation of government policies for eHealth adoption. Additionally, there should be policies and processes that govern dictionary management, as this has been found to be very critical in eHealth adoption (citation 9 should be added).

Ethical and Legal Dimension: Ethical and legal considerations must be observed while implementing eHealth more so since eHealth involves the processing and use of patient data. As reported in other studies [6], [7], ethical and legal issues have been found to play an important part in the successful adoption of eHealth.

Finance Dimension: The successful adoption of eHealth requires adequate financial support for implementation, operation, user training, awareness, and maintenance. Like this study, several studies [8]-[10] underscore the relative importance of adequate finances in boosting eHealth adoption. Adequate financial resources enable the procurement, installation and maintenance of digital technologies. Users can be motivated financially [11] which increases their desire to use digital health systems.

\section{Conclusion}

The study shows that there are several frameworks that have been used to examine eHealth adoption in developing countries. These include; Technology Acceptance Model Unified Theory of Acceptance and Use of Technology, Organisation and Environment framework the Human Organisation Technology Fit, Extended Technology Acceptance Model, Diffusion Innovation Theory, Logic model, Theory of Planned Behaviour, Theory of Reasoned Act, Fit between Individuals Task and Technology and Patient m-Health Adoption. Technology Acceptance Model, Unified Theory of Acceptance and Use of Technology and Technology, Organisation and Environment are the most widely adopted frameworks. However, other researchers equally report critical success factors for eHealth adoption yet they are not addressed in these frameworks. Hence, a more comprehensive framework that integrates all factors has been developed to capture the salient features for successful adoption of eHealth in developing countries. This framework proposes eight key dimensions namely, Socio-demographic, Technology, Information, Sociocultural, Organization, Governance, Ethical and legal, and Financial dimensions. Future research will involve the implementation of the eight (8)-dimension eHealth adoption framework, as proposed by this research, to validate its applicability in the developing world context.

\section{ACKNOWLEDGEMENTS}

Sincere gratitude goes out to the Government of Uganda, through the Makerere University, Research and Innovations Fund for funding this research. Similarly, appreciation goes out to all the authors and research assistants for their relentless effort and perseverance to have this review successfully done.

\section{REFERENCES}

[1] Slater, H., Dear, B. F., Merolli, M. A., Li, L. C., \& Briggs, A. M. (2016). Use of eHealth technologies to enable the implementation of musculoskeletal Models of Care: Evidence and practice. Best Practice \& Research Clinical Rheumatology, 30(3), 483-502. 
Health Informatics - An International Journal (HIIJ) Vol.10, No.3, August 2021

[2] Barbabella, F., Melchiorre, M. G., Quattrini, S., Papa, R., Lamura, G., Richardson, E., \& van Ginneken, E. (2017). How can eHealth improve care for people with multimorbidity in Europe? Copenhagen: World Health Organization, Regional Office for Europe.

[3] Ross, J., Stevenson, F., Lau, R., \& Murray, E. (2016). Factors that influence the implementation of ehealth: a systematic review of systematic reviews (an update). Implementation science, 11(1).

[4] Kiberu, V. M., Mars, M., \& Scott, R. E. (2017). Barriers and opportunities to implementation of sustainable e-Health programmes in Uganda: A literature review. African journal of primary health care \& family medicine, 9(1), 1-10.

[5] Funmi Adebesin, Paula Kotzé, Darelle van Greunen, Rosemary Foster (2013) Barriers and Challenges to the Adoption of E-Health Standards in Africa. (2013). Health Informatics South Africa (HISA) conference, Port Elizabeth, 3-5 July 2013

[6] Ogoe, H. A., Asamani, J. A., Hochheiser, H., \& Douglas, G. P. (2018). Assessing Ghana's eHealth workforce: implications for planning and training. Human resources for health, 16(1), 1-11.

[7] Hersh, W., Margolis, A., Quirós, F., \& Otero, P. (2010). Building a health informatics workforce in developing countries. Health Affairs, 29(2), 274-277.

[8] Ashaba, J., \& Nabukenya, J. (2020). Assessing Evaluation of eHealth Interventions in Uganda: Practices, Challenges and Insights. Journal of Health Informatics in Africa, 7(1), 12-23.

[9] Keny, A., Wanyee, S., Kwaro, D., Mulwa, E., \& Were, M. C. (2015). Developing a national-level concept dictionary for EHR implementations in Kenya.

[10] Omaswa C. Ministry of Health: Uganda National eHealth policy [homepage on the Internet]. 2013 [cited 2016 Dec 9]. Available from: http://library.health.go.ug/download/file/fid/580685

[11] Alaboudi, A., Atkins, A., Sharp, B., Balkhair, A., Alzahrani, M., \& Sunbul, T. (2016). Barriers and challenges in adopting Saudi telemedicine network: The perceptions of decision makers of healthcare facilities in Saudi Arabia. Journal of infection and public health, 9(6), 725-733.

[12] Tsiknakis, M., \& Kouroubali, A. (2009). Organizational factors affecting successful adoption of innovative eHealth services: A case study employing the FITT framework. International journal of medical informatics, 78(1), 39-52.

[13] Hossain, N., Yokota, F., Sultana, N. and Ahmed, A. (2018) Factors Influencing Rural End-Users' Acceptance of e-Health in Developing Countries: A Study on Portable Health Clinic in Bangladesh. Telemedicine and e-Health, 25, 221-229.

[14] Feijt, M. A., de Kort, Y. A., Bongers, I. M., \& IJsselsteijn, W. A. (2018). Perceived drivers and barriers to the adoption of emental health by psychologists: the construction of the levels of adoption of emental health model. Journal of medical Internet research, 20(4), e153.

[15] Tavares, J., \& Oliveira, T. (2017). Electronic health record portal adoption: a cross country analysis. BMC medical informatics and decision making, 17(1), 1-17.

[16] Kitchenham, B., \& Brereton, P. (2013). A systematic review of systematic review process research in software engineering. Information and software technology, 55(12), 2049-2075.

[17] Domínguez-Mayo, F. J., Escalona, M. J., Mejías, M., Aragón, G., García-García, J. A., Torres, J., \& Enríquez, J. G. (2015). A strategic study about quality characteristics in e-health systems based on a systematic literature review. The Scientific World Journal, 2015.

[18] AlBar, A. M., \& Hoque, M. R. (2019). Patient acceptance of e-health services in Saudi Arabia: an integrative perspective. Telemedicine and e-Health, 25(9), 847-852.

[19] Hoque, M. R., \& Bao, Y. (2015). Cultural influence on adoption and use of e-Health: evidence in Bangladesh. Telemedicine and e-Health, 21(10), 845-851.

[20] Adenuga, K. I., Iahad, N. A., \& Miskon, S. (2017). Towards reinforcing telemedicine adoption amongst clinicians in Nigeria. International journal of medical informatics, 104, 84-96.

[21] Hossain, M. N., Okajima, H., Kitaoka, H., \& Ahmed, A. (2017). Consumer acceptance of eHealth among rural inhabitants in developing countries (A Study on Portable Health Clinic in Bangladesh). Procedia computer science, 111, 471-478.

[22] Rahimi, B., Nadri, H., Afshar, H. L., \& Timpka, T. (2018). A systematic review of the technology acceptance model in health informatics. Applied clinical informatics, 9(3), 604.

[23] Alsisi, E. A., Al-Ashaab, A., \& Abualfaraa, W. A. (2020). The Development of a Smart Health Awareness Message Framework Based on the Use of Social Media: Quantitative Study. Journal of medical Internet research, 22(7), e16212.

[24] Hoque, M. R. (2016). An empirical study of mHealth adoption in a developing country: the moderating effect of gender concern. BMC medical informatics and decision making, 16(1), 1-10. 
Health Informatics - An International Journal (HIIJ) Vol.10, No.3, August 2021

[25] Touray, A., Salminen, A., \& Mursu, A. (2015). Internet adoption at the user level: empirical evidence from the Gambia. Information Technology for Development, 21(2), 281-296.

[26] Addotey-Delove, M. N. A., Scott, R. E., \& Mars, M. (2020). Review of patients perspectives of mhealth adoption factors in the developing world. Development of a framework.

[27] Nilashi, M., Ahmadi, H., Ahani, A., Ravangard, R., \& bin Ibrahim, O. (2016). Determining the importance of hospital information system adoption factors using fuzzy analytic network process (ANP). Technological Forecasting and Social Change, 111, 244-264.

[28] Haneem, F., Kama, N., Taskin, N., Pauleen, D., \& Bakar, N. A. A. (2019). Determinants of master data management adoption by local government organizations: An empirical study. International Journal of Information Management, 45, 25-43.

[29] Shahzad, K., Jianqiu, Z., Zia, M. A., Shaheen, A., \& Sardar, T. (2021). Essential factors for adopting hospital information system: a case study from Pakistan. International Journal of Computers and Applications, 43(1), 26-37.

[30] Baryashaba, A., Musimenta, A., Mugisha, S., \& Binamungu, L. P. (2019, May). Investigating the adoption of an integrated hospital information system in rural Uganda: A case of Kisiizi Hospital. In International Conference on Social Implications of Computers in Developing Countries (pp. 316325). Springer, Cham.

[31] Nassoura, A. B. (2020). Critical Success Factors For Adoption Of Cloud Computing In Jordanian Healthcare Organizations. International Journal of Scientific \& Technology Research, 9(4), 27982803.

[32] Saengchaia, S., Pattanapongthornb, J., \& Jermsittiparsertc, K. (2019). The Role of Subjective Norms on the Adoption of Information and Communication Technology in Health Care in Thailand. International Journal of Innovation, Creativity and Change, 8(8), 256-276.

[33] Popela, I., Zuva, T., \& Appiah, M. (2019, November). Factors That Influence the Adoption of Electronic Patients Records Management Systems in South Africa. In 2019 International Multidisciplinary Information Technology and Engineering Conference (IMITEC) (pp. 1-9). IEEE.

[34] Mukred, M., Yusof, Z. M., Mokhtar, U. A., Sadiq, A. S., Hawash, B., \& Ahmed, W. A. (2021). Improving the decision-making process in the higher learning institutions via electronic records management system adoption.

[35] Shahzad, K., Jianqiu, Z., Zubedi, A., Xin, W., Wang, L., \& Hashim, M. (2020). DANP-based method for determining the adoption of hospital information system. International Journal of Computer Applications in Technology, 62(1), 57-70.

[36] Mauco, K. L., Scott, R. E., \& Mars, M. (2019). Development of an eHealth readiness assessment framework for Botswana and other developing countries: interview study. JMIR medical informatics, 7(3), e12949.

[37] Charness, N., \& Boot, W. R. (2016). Technology, gaming, and social networking. In Handbook of the Psychology of Aging (pp. 389-407). Academic Press.

[38] Baker, J. (2012). The technology-organization-environment framework. Information systems theory, 231-245. 


\section{AUTHORS}

Agnes Rwashana Semwanga is an Associate Professor of Information Systems and the Deputy Principal of the College of Computing and Information Sciences at Makerere University. She teaches and supervises graduate students on the Master of Health Informatics programme, has done extensive research, published and developed System Dynamics models of health issues including immunisation, maternal health, neonatal health and food security.

Hasifah is attached to the Department of Information Systems at College of Computing and Information Sciences at Makerere University, where she has served for 10 years as a professional career researcher and lecturer. She is an interdisciplinary design science researcher and information systems technologist specializing in database systems, systems dynamics and health informatics.

Swaib Kyanda Kaawaase is a lecturer of wireless communication networks. He is the head of networks department at the College of Computing and Information Sciences at Makerere University. He is a researcher with passion on the use of ICT for the wellbeing of human life in all forms.

Mark is an active researcher in the field of data science, natural language processing and machine learning in general. He is particularly interested in practical applications of data science and machine learning in finding novel solutions to various problem areas like health, nature conservation, agriculture, urban planning and energy.
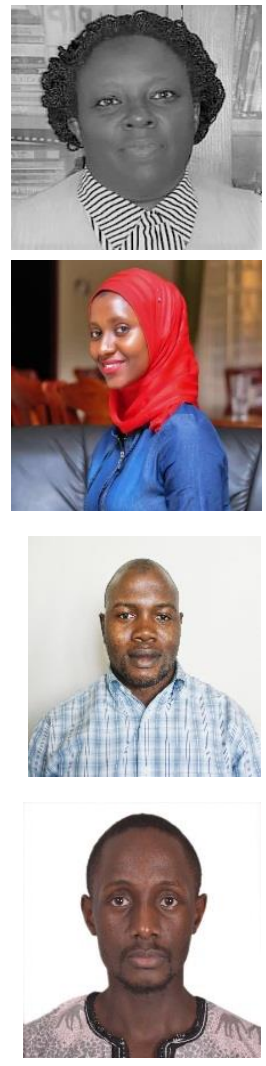